\title{
A FIXED POINT THEOREM IN MENGER SPACE
}

\author{
${ }^{1}$ B. D. Pant, ${ }^{2}$ Sunny Chauhan* \\ ${ }^{1}$ Government Degree College, Champawat, Uttarakhand, India. \\ ${ }^{2}$ Department of Applied Science, Bhagwant Institute of Technology, Bhagwantpuram, Muzaffar \\ Nagar, 251315, Uttar Pradesh, India. \\ *Corresponding address: sun.gkv@gmail.com \\ Received 28 May, 2010; Revised 19 November, 2010
}

\begin{abstract}
The purpose of this paper is to introduce the concept of weakly compatible maps in Menger space and prove a common fixed point theorem in this space.
\end{abstract}

\section{Mathematics Subject Classification: 54H25.}

Keywords: Triangle norm; Menger space; Fixed point theorem; Weakly compatible maps.

\section{INTRODUCTION AND PRELIMINARIES}

K. Menger [4] introduced the notion of a probabilistic metric space in 1942 and since then, the theory of probabilistic metric spaces has developed in many directions, especially, in nonlinear analysis and applications [1]. The idea of Menger was to use distribution functions instead of nonnegative real numbers as values of the metric. Schweizer and Sklar [7] studied this concept and gave some fundamental results on this space. The important development of fixed point theory in Menger spaces was due to Sehgal and Bharucha-Reid [8].

Jungck [2] introduced the concept of compatible maps. And this condition has further been weakened by introducing the notion of weakly compatible mappings by Jungck and Rhoades [3]. The concept of weakly compatible mappings is most general as each pair of compatible mappings is weakly compatible but the reverse is not true. Recently in this line, Singh and Jain [10] introduced the notion of weakly compatible maps in Menger space to establish a common fixed point theorem.

In this paper we establish a common fixed point theorem for two pairs of weakly compatible mappings in Menger space without appeal to continuity. First we recall some definitions and known results in Menger spaces.

Definition 1.1. A triangular norm $T$ (shortly $t$-norm) is a binary operation on the unit interval $[0,1]$ such that for all $a, b, c, d \in[0,1]$ the following conditions are satisfied:

(i) $T(a, \mathbf{1})=a$;

(ii) $T(a, b)=T(b, a)$; 
(iii) $T(a, b) \leq T(c, d)$ whenever $a \leq c$ and $b \leq d$;

(iv) $T(a, T(b, c))=T(T(a, b), c)$;

Two typical examples of continuous $t$-norm are $T(a, b)=a b$ and $T(a, b)=\min \{a, b\}$. Now ${ }^{t}$-norms are recursively defined by $T^{\mathbf{1}}=T$ and

$$
T^{n}\left(x_{1}, \ldots \ldots, x_{n+1}\right)=T\left(T^{n-1}\left(x_{1}, \ldots \ldots, x_{n}\right), x_{n+1}\right)
$$

for $n \geq 2$ and $x_{i} \in[0,1]$, for all $i \in\{1,2, \ldots, n+1\}$.

Definition 1.2. A mapping $F: R \rightarrow R^{+}$is said to be a distribution function if it is non-decreasing and left continuous with infimun $\{F(t): t \in R\}=\mathbf{0}$ and suprimun $\{F(t): t \in R\}=\mathbf{1}$.

We will denote by $\boldsymbol{\Delta}_{+}$the family of all distribution function on $[-\infty, \infty]$. A special element of $D_{+}$(where $D_{+} \subseteq \Delta_{+}$) is the function $\varepsilon_{0}$ defined by

$$
\varepsilon_{0}(t)= \begin{cases}0, & \text { if } t \leq 0 \\ 1, & \text { if } t>0\end{cases}
$$

If $X$ is a non-empty set $F: X \times X \rightarrow D_{+}$is called a probabilistic distance on $X$ and $F(x, y)$ is usually denoted by $F_{x, y}$.

Definition 1.3. The ordered pair $(X, F)$ is called a probabilistic metric space (shortly PM-space) if $X$ is a non-empty set and $F$ is a probabilistic distance satisfying the following conditions: for all $x, y, z \in X$ and $t, s>\mathbf{0}$,

(i) $F_{x, y}(t)=\mathbf{1} \Leftrightarrow x=y$;

(ii) $F_{x, y}(\mathbf{0})=\mathbf{0}$;

(iii) $F_{x, y}(t)=F_{y, x}(t)$;

(iv) $F_{x, z}(t)=\mathbf{1}, F_{z, y}(s)=\mathbf{1} \Rightarrow F_{x, y}(t+s)=\mathbf{1}$.

The ordered triple $(X, F, T)$ is called a Menger space if $(X, F)$ is a PM-space, $T$ is a $t$-norm and the following inequality hold:

(v) $F_{x, y}(t+s) \geq T\left(F_{x, z}(t), F_{z, y}(s)\right)$.

Definition 1.4. Let $(X, F, T)$ be a Menger space and ${ }^{T}$ be a continuous ${ }^{t}$-norm.

(i) A sequence $\left\{x_{n}\right\}$ in $X$ is said to be converge to a point $X$ in $X$ iff for every $\varepsilon>0$ and $\lambda \in(0,1)$, there exists an integer $N$ such that $F_{x_{n}, x}(\varepsilon)>1-\lambda$ for all $n \geq N$.

(ii) A sequence $\left\{x_{n}\right\}$ in $X$ is said to be Cauchy if for every $\varepsilon>\mathbf{0}$ and $\lambda \in(0,1)$, there exists an integer $N$ such that $F_{x_{n}, x_{m}}(\varepsilon)>1-\lambda$ for all $n, m \geq N$. 
(iii) A Menger space in which every Cauchy sequence is convergent is said to be complete.

Definition 1.5 [5]. Self maps $A$ and $B$ of a Menger space $(X, F, T)$ are said to be compatible if $F_{A B x_{n}, B A x_{n}}(t) \rightarrow \varepsilon_{0}(t)$ for all $t>\mathbf{0}$, whenever $\left\{x_{n}\right\}$ is a sequence in $X$ such that $A x_{n}, B x_{n} \rightarrow X$ for some $x$ in $X$ as $n \rightarrow \infty$.

Definition 1.6 [10]. Self maps $A$ and $B$ a Menger space $(X, F, T)$ are said to be weakly compatible (or coincidentally commuting) if they commute at their coincidence points, that is if $A x=B x$ for some $x \in X$ then $A B x=B A x$.

Proposition 1.7 [10]. If self maps $A$ and $B$ of a Menger space $(X, F, T)$ are compatible then they are weakly compatible.

The following is an example of pair of self maps in a Menger space which are weakly compatible but not compatible.

Example 1.8. Let $(X, d)$ be a metric space where $X=[0,1]$ and $(X, F, T)$ be the induced Menger space with $F_{x, y}(t)=\frac{t}{t+d(x, y)}$, for all $x, y \in X$ and $t>\mathbf{0}$. We define self maps $A$ and $B$ as follows:

$$
A(x)=\left\{\begin{array}{ll}
1-x, & \text { if } 0 \leq x<\frac{\mathbf{1}}{\mathbf{2}} ; \\
1, & \text { if } \frac{\mathbf{1}}{\mathbf{2}} \leq x \leq 1 .
\end{array} \text { and } \quad B(x)= \begin{cases}x, & \text { if } 0 \leq x<\frac{\mathbf{1}}{\mathbf{2}} \\
1, & \text { if } \frac{\mathbf{1}}{\mathbf{2}} \leq x \leq 1 .\end{cases}\right.
$$

Taking $x_{n}=\frac{1}{2}-\frac{1}{n}$. We get $A x_{n}=\frac{1}{2}+\frac{1}{n}, B x_{n}=\frac{1}{2}-\frac{1}{n}$. Thus, $A x_{n} \rightarrow \frac{1}{2} B x_{n} \rightarrow \frac{1}{2}$.

Hence $=\frac{\mathbf{1}}{\mathbf{2}}$. Further $A B x_{n}=\frac{\mathbf{1}}{\mathbf{2}}+\frac{\mathbf{1}}{n}, B A x_{n}=\mathbf{1}$.

Now; $\lim _{n \rightarrow \infty} F_{A B x_{n}, B A x_{n}}(t)=\lim _{n \rightarrow \infty} F_{\frac{1}{2}+\frac{1}{n}, 1}(t)=\frac{t}{t+\frac{1}{2}}=\frac{2 t}{2 t+1}<1$, for all $t>0$.

Hence $(A, B)$ is not compatible.

Coincidence points of $A$ and $B$ are in $\left[\frac{\mathbf{1}}{\mathbf{2}}, 1\right]$. Now for any $x \in\left[\frac{\mathbf{1}}{\mathbf{2}}, 1\right] . A x=B x=\mathbf{1}$ and $A B(x)=A(\mathbf{1})=1=B(\mathbf{1})=B A(x)$. Thus $(A, B)$ is weakly compatible

Lemma 1.9 [6, 9]. Let $(X, F, T)$ be a Menger space and define $E_{\lambda, F}: X^{2} \rightarrow R^{+} \cup\{0\}$ by

$$
E_{\lambda, F}(x, y)=\inf \left\{t>0: F_{x, y}(t)>1-\lambda\right\}
$$

for each $\lambda \in(0,1)$ and $x, y \in X$. Then we have 
(i) For any $\mu \in(0,1)$ there exists $\lambda \in(0,1)$ such that

$$
E_{\mu, F}(x, z)<\varepsilon_{\lambda, F}(x, y)+E_{\lambda, F}(y, z)
$$

$$
\text { any } x, y, z \in X \text {; }
$$

(ii) The sequence $\left\{x_{n}\right\}_{n \in N}$ is convergent with respect to Menger probabilistic metric $F$ if and only if $E_{\lambda, F}\left(x_{n}, x\right) \rightarrow \mathbf{0}$. Also the sequence $\left\{x_{n} \boldsymbol{1}_{n \in N}\right.$ is Cauchy sequence with respect to Menger probabilistic metric $F$ if and only if it is a Cauchy sequence with $E_{\lambda, F}$.

Lemma 1.10 [5]. Let $(X, F, T)$ be a Menger space. If there exists a constant $k \in(0,1)$ such that

$$
F_{x, y}(k t) \geq F_{x, y}(t)
$$

for all $x, y \in X$ and $t>0$ then $x=y$.

\section{RESULTS}

Theorem 2.1. Let $A, L, M$ and $S$ be self maps on a complete Menger space $(X, F, T)$ and suppose the following are satisfied:

(i) $L(X) \subseteq S(X), M(X) \subseteq A(X)$;

(ii) $S(X)$ and $A(X)$ are complete subspace of $X$.

(iii)

$$
\begin{gathered}
F_{L x, M y}(k t) \geq \min \left\{\begin{array}{c}
F_{A x, L x}(t), F_{S y, M y}(t), F_{S y, L x}(\alpha t), F_{A x, M y}((2-\alpha) t), \\
F_{A x, S y}(t)
\end{array}\right\} \\
\text { for all } x, y \in X, k \in(0,1), \alpha \in(0,2) \text { and } t>\mathbf{0} ;
\end{gathered}
$$

(iv) The pairs $(L, A)$ and $(M, S)$ are weakly compatible;

In addition assume that

$$
E_{\lambda, F}(x, y)=\inf \left\{t>0: F_{x, y}(t)>1-\lambda\right\}
$$

for each $\lambda \in(0,1)$ and $x, y \in X$.

Then $A, L, M$ and $S$ have a unique common fixed point.

Proof. Let $x_{0}, x_{1}, \quad x_{2} \in X$ be such that $L x_{0}=S x_{1}=y_{0}$ and $M x_{1}=A x_{2}=y_{2}$. Inductively, we construct sequences $\left\{x_{n}\right\}$ and $\left\{y_{n}\right\}$ in $X$ such that $L x_{2 n}=S x_{2 n+1}=y_{2 n}$ and $M x_{2 n+1}=A x_{2 n+2}=y_{2 n+1}$ for $n=0,1,2,3,4 \ldots \ldots \ldots \ldots \ldots$.

Putting $x=x_{2 n}$ and $y=x_{2 n+1}$ for $t>0$ and $\alpha=1-q$ with $q \in(0,1)$ in (iii), we get

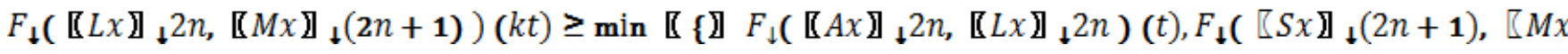

$\left.F_{\downarrow}\left(\llbracket A x \rrbracket_{\downarrow} 2 n, \llbracket M x \rrbracket_{\downarrow}(2 n+1)\right)((1+q) t), F_{\downarrow}\left(\llbracket A x \rrbracket_{\downarrow} 2 n, \llbracket S x \rrbracket{ }_{\downarrow}(2 n+1)\right)(t)\right\}$ 


$$
\begin{aligned}
F_{y_{2 n}, y_{2 n+1}}(k t) \geq & \min \left\{F_{y_{2 n-1}, y_{2 n}}(t), F_{y_{2 n}, y_{2 n+1}}(t), 1, F_{y_{2 n-1}, y_{2 n+1}}((1+q) t), F_{y_{2 n-1}, y_{2 n}}(t)\right\} \\
& \geq \min \left\{F_{y_{2 n-1}, y_{2 n}}(t), F_{y_{2 n}, y_{2 n+1}}(t), F_{y_{2 n-1}, y_{2 n}}(t), F_{y_{2 n}, y_{2 n+1}}(q t)\right\} \\
& \geq \min \left\{F_{y_{2 n-1}, y_{2 n}}(t), F_{y_{2 n}, y_{2 n+1}}(t), F_{y_{2 n}, y_{2 n+1}}(q t)\right\}
\end{aligned}
$$

Letting $q \rightarrow \mathbf{1}$, we get

$$
\begin{aligned}
F_{y_{2 n}, y_{2 n+1}}(k t) & \geq \min \left\{F_{y_{2 n-1}, y_{2 n}}(t), F_{y_{2 n}, y_{2 n+1}}(t), F_{y_{2 n}, y_{2 n+1}}(t)\right\} \\
& =\min \left\{F_{y_{2 n-1}, y_{2 n}}(t), F_{y_{2 n}, y_{2 n+1}}(t)\right\} .
\end{aligned}
$$

Hence

$$
F_{y_{2 n}, y_{2 n+1}}(k t) \geq \min \left\{F_{y_{2 n-1}, y_{2 n}}(t), F_{y_{2 n}, y_{2 n+1}}(t)\right\} .
$$

Similarly,

$$
F_{y_{2 n+1}, y_{2 n+2}}(k t) \geq \min \left\{F_{y_{2 n}, y_{2 n+1}}(t), F_{y_{2 n+1}, y_{2 n+2}}(t)\right\} \text {. }
$$

Therefore, for all $n$ we have:

$$
F_{y_{n}, y_{n+1}}(k t) \geq \min \left\{F_{y_{n-1}, y_{n}}(t), F_{y_{n}, y_{n+1}}(t)\right\}
$$

Consequently,

$$
F_{y_{n}, y_{n+1}}(t) \geq \min \left\{F_{y_{n-1}, y_{n}}\left(k^{-1} t\right), F_{y_{n}, y_{n+1}}\left(k^{-1} t\right)\right\}
$$

By repeated application of above inequality, we get for each $j \in\{1,2,3, \ldots \ldots\}$.

$$
\begin{aligned}
F_{y_{n}, y_{n+1}}(t) & \geq \min \left\{F_{y_{n-1}, y_{n}}\left(k^{-1} t\right), F_{y_{n-1}, y_{n}}\left(k^{-2} t\right), F_{y_{n}, y_{n+1}}\left(k^{-2} t\right)\right\} \\
& =\min \left\{F_{y_{n-1}, y_{n}}\left(k^{-1} t\right), F_{y_{n}, y_{n+1}}\left(k^{-2} t\right)\right\} \\
& \geq \ldots \ldots \ldots \ldots \geq \min \left\{F_{y_{n-1}, y_{n}}\left(k^{-1} t\right), F_{y_{n}, y_{n+1}}\left(k^{-j} t\right)\right\}
\end{aligned}
$$

and so for each $\lambda \in(0,1)$ we have

$$
\begin{aligned}
& E_{\lambda, F}\left(y_{n}, y_{n+1}\right)=\inf \left\{t>0: F_{y_{n}, y_{n+1}}(t)>1-\lambda\right\} \\
& \leq \inf \{t>0 \text { : } \\
& \left.\llbracket \min \rrbracket\left\{F_{\downarrow}\left(y_{\downarrow}(n-\mathbf{1}), y_{\downarrow} n\right)\left(k^{\boldsymbol{\top}}(-\mathbf{1}) t\right), F_{\downarrow}\left(y_{\downarrow} n, y_{\downarrow}(n+\mathbf{1})\right)\left(k^{\boldsymbol{\top}}(-j) t\right)\right\}>\mathbf{1}-\lambda\right\}
\end{aligned}
$$




$$
\begin{aligned}
& \quad \leq \max \left\{\inf \left\{t>0: F_{\downarrow}\left(y_{\downarrow}(n-\mathbf{1}), y_{\downarrow} n\right)\left(k^{\uparrow}(-\mathbf{1}) t\right)>\mathbf{1}-\lambda\right\}, \inf \{t>0:\right. \\
& \left.\left.F_{\downarrow}\left(y_{\downarrow} n, y_{\downarrow}(n+\mathbf{1})\right)\left(k^{\uparrow}(-j) t\right)>\mathbf{1}-\lambda\right\}\right\} \\
& \quad \leq \max \left\{k E_{\downarrow}(\lambda, F)\left(y_{\downarrow}(n-\mathbf{1}), y_{\downarrow} n\right), k^{\mathbf{\top} j} E_{\downarrow}(\lambda, F)\left(y_{\downarrow} n, y_{\downarrow}(n+\mathbf{1})\right)\right\} .
\end{aligned}
$$

Since, $k^{j} E_{\lambda, F}\left(y_{n}, y_{n+1}\right) \rightarrow \mathbf{0}$ as $j \rightarrow \infty$, it follows that:

$$
E_{\lambda, F}\left(y_{n}, y_{n+1}\right) \leq k \quad E_{\lambda, F}\left(y_{n-1}, y_{n}\right) \leq k^{n} E_{\lambda, F}\left(y_{0}, y_{1}\right) \text { for every } \lambda \in(0,1) \text {. }
$$

Now, we show that $\left\{y_{n}\right\}$ is a Cauchy sequence. For every $\mu \in(0,1)$, there exists $\gamma \in(0,1)$ such that, for $m \geq n$,

$$
\begin{aligned}
E_{\mu, F}\left(y_{n}, y_{m}\right) & \leq E_{\gamma, F}\left(y_{m-1}, y_{m}\right)+E_{\gamma, F}\left(y_{m-2}, y_{m-1}\right)+\cdots \ldots \ldots \ldots \ldots+E_{\gamma, F}\left(y_{n}, y_{n+1}\right) \\
& \leq E_{\gamma, F}\left(y_{0}, y_{1}\right) \sum_{i=n}^{m-1} k^{i} \rightarrow \mathbf{0}
\end{aligned}
$$

as $m, n \rightarrow \infty$. Hence by Lemma $1.9,\left\{y_{n}\right\}$ is a Cauchy sequence in $X$. Since $X$ is complete then $\left\{y_{n}\right\}$ converges to $z \in X$. That is

$\lim _{n \rightarrow \infty} y_{n}=\lim _{n \rightarrow \infty} L x_{2 n}=\lim _{n \rightarrow \infty} M x_{2 n+1}=\lim _{n \rightarrow \infty} S x_{2 n+1}=\lim _{n \rightarrow \infty} A x_{2 n}=z$.

Since $L(X) \subseteq S(X)$ and $M(X) \subseteq A(X)$ then there exist, $v, w \in X$ such that $S(v)=z$ and $A(w)=z$. Now we prove that $S(v)=M(v)=z$ and $A(w)=L(w)=z$.

Put $x=x_{2 n}, y=v$ and $\alpha=\mathbf{1}$ in (iii), then we get

$F_{L x_{2 n}, M v}(k t) \geq \min \square\left\{F_{A x_{2 n}, L x_{2 n}}(t), F_{S v, M v}(k t), F_{S v, L x_{2 n}}(t), F_{A x_{2 n}, M v}(t), F_{A x_{2 n}, S v}(t)\right\}$

as $n \rightarrow \infty$, we have

$F_{z, M v}(k t) \geq \min \left\{F_{z, z}(t), F_{z, M v}(t), F_{z, z}(t), F_{z, M v}(t), F_{z, z}(t)\right\}$

then on simplification

$F_{z, M v}(k t) \geq F_{z, M v}(t)$

Thus, by Lemma $1.10, z=M v$. Therefore, $M(v)=S(v)=z$.

Now put $x=w, y=x_{2 n+1}$ and $\alpha=\mathbf{1}$ in (iii), then we get

$F_{L W, M x_{\mathbf{2 n + 1}}}(k t) \geq \min \left\{\begin{array}{c}F_{A w, L w}(t), F_{S x_{\mathbf{2 n + 1}}, M x_{\mathbf{2 n + 1}}}(t), F_{S x_{\mathbf{2} n+\mathbf{1}}, L W}(t), F_{A w, M x_{\mathbf{2} n+\mathbf{1}}}(t), \\ F_{A w, S x_{\mathbf{2} n+\mathbf{1}}}(t)\end{array}\right\}$

as $n \rightarrow \infty$, we have 
$F_{L w, z}(k t) \geq \min \left\{F_{z, L w}(t), F_{z, z}(t), F_{z, L w}(t), F_{z, z}(t), F_{z, z}(t)\right\}$

then on simplification

$F_{L w, z}(k t) \geq F_{L w, z}(t)$

Thus, by Lemma $1.10, z=L w$. Therefore $L w=A w=z$.

Also it is given that the pairs $(L, A)$ and $(M, S)$ are weakly compatible, then $L(A(w))=A(L(w))$, that is $L z=A z$ and $M(S(v))=S(M(v))$, that is $M z=S z$. Now we prove that $L z=A z=z$ and $M z=S z=z$.

Put $x=z, y=x_{2 n+1}$ and $\alpha=1$ in (iii), then we get

$F_{L z, M x_{2 n+1}}(k t) \geq \min \left\{\begin{array}{c}F_{A z, L z}(t), F_{S x_{2 n+1}, M x_{2 n+1}}(t), F_{S x_{2 n+1}, L z}(t), F_{A z, M x_{2 n+1}}(t), \\ F_{A z, S x_{2 n+1}}(t)\end{array}\right\}$

as $n \rightarrow \infty$, we have

$F_{A z, z}(k t) \geq \min \left\{F_{A z, A z}(t), F_{z, z}(t), F_{z, A z}(t), F_{A z, z}(t) F_{A z, z}(t)\right\}$

then on simplification, we have

$F_{A z, z}(k t) \geq F_{A z, z}(t)$

Thus, by Lemma $1.10, z=A z$. Therefore, $z=L z=A z$.

Now put $x=x_{\mathbf{2 n}}, y=z$ and $\alpha=\mathbf{1}$ in (iii), then we get

$F_{L x_{\mathbf{2}}, M z}(k t) \geq \min \left\{\begin{array}{c}F_{A x_{2 n}, L x_{2 n}}(t), F_{S z, M z}(t), F_{S z, L x_{2 n}}(t), F_{A x_{2 n}, M z}(t), \\ F_{A x_{2 n}, S z}(t)\end{array}\right\}$

as $n \rightarrow \infty$, we have

$F_{z, S Z}(k t) \geq \min \left\{F_{z, z}(t), F_{S z, S z}(t), F_{S z, z}(t), F_{z, S z}(t), F_{z, S z}(t)\right\}$

then on simplification

$F_{z, S z}(k t) \geq F_{z, S z}(t)$

Thus, by Lemma $1.10, z=S z$. Therefore, $z=M z=S z$. Now, combine all the results it is clear that $z=A z=L Z=M z=S z$. That is $Z$ is the common fixed point.

Uniqueness: Let $u(u \neq z)$ be another common fixed point of $A, L, M$ and $S$. Taking $x=z$, $y=u$ and $\alpha=\mathbf{1}$ in (iii), then we get

$\left.F_{L z, M u}(k t) \geq \min \llbracket\{\square] F_{A z, L z}(t), F_{S u, M u}(t), F_{S u, L z}(t), F_{A z, M u}(t), F_{A z, S u}(t)\right\}$ 
then on simplification

$$
F_{z, u}(k t) \geq F_{z, u}(t)
$$

Thus, by Lemma $1.10, z=u$ and so the uniqueness of the common fixed point.

The following example illustrates Theorem 2.1.

Example 2.2. Let $X=[0,30)$ with the metric $d$ defined by $d(x, y)=|x-y|$ and for each $t \in[0,1]$ define

$$
F_{x, y}(t)=\left\{\begin{array}{cl}
\frac{t}{t+|x-y|}, & \text { if } t>0, \\
0, & \text { if } t=0 .
\end{array}\right.
$$

for all $x, y \in X$. Clearly $(X, F, T)$ is a complete Menger space. Where $T$ is a continuous $t$ norm. Define $A, L, M$ and $S: X \rightarrow X$ by

$$
\begin{gathered}
A(X)=\left\{\begin{array}{rl}
0, & \text { if } x=0, \\
12, & \text { if } 0<x<15, \\
x-9, & \text { if } 15<x<30 .
\end{array} \quad S(X)=\left\{\begin{array}{cc}
0, & \text { if } x=0, \\
6, & \text { if } 0<x<15, \\
x-6, & \text { if } 15<x<30 .
\end{array}\right.\right. \\
L(X)=\left\{\begin{array}{ll}
0, & \text { if } x=0, \\
6, & \text { if } 0<x<30 .
\end{array} \quad M(X)= \begin{cases}0, & \text { if } x=0, \\
9, & \text { if } 0<x<30 .\end{cases} \right.
\end{gathered}
$$

Then $A, L, M$ and $S$ satisfy all the conditions of Theorem 2.1 with $k \in(0,1)$ and have a unique common fixed point $0 \in X$. It may be noted in this example that the mappings $L$ and $A$ commute at coincidence point $0 \in X$. So $L$ and $A$ are weakly compatible maps. Similarly, $M$ and $S$ are weakly compatible maps. To see the pairs $(L, A)$ and $(M, S)$ are not compatible. Let us consider a sequence $\left\{x_{n}\right\}$ defined as $x_{n}=15+\frac{\mathbf{1}}{n}, n \geq \mathbf{1}$, then $x_{n} \rightarrow 15$ as $n \rightarrow \infty$. Then $\lim _{n \rightarrow \infty} L x_{n}=\mathbf{6} \lim _{n \rightarrow \infty} A x_{n}=\mathbf{6} \operatorname{but}_{n \rightarrow \infty} \lim _{L A x_{n}, A L x_{n}}(t)=\frac{t}{t+|6-12|} \neq \mathbf{1}$. Thus the pair $(L, A)$ is not compatible. Also $\lim _{n \rightarrow \infty} M x_{n}=\mathbf{9} \lim _{n \rightarrow \infty} S x_{n}=\mathbf{9}$ but $\lim _{n \rightarrow \infty} F_{M S x_{n}, S M x_{n}}(t)=\frac{t}{t+|9-\mathbf{6}|} \neq \mathbf{1}$. So the pair $(M, S)$ is not compatible. All the mappings involved in this example are discontinuous even at the common fixed point $x=\mathbf{0}$.

\section{REFERENCES}

[1] Chang S S, Lee B S, Cho Y J, Chen Y Q, Kang S M \& Jung J S, Generalized contraction mapping principles and differential equations in probabilistic metric spaces, Proc. Amer. Math. Soc. 124 (1996), 2367. 
[2] Jungck G, Compatible mappings and common fixed points, Internat. J. Math. \& Math. Sci. 9 (1986), 771.

[3] Jungck $G$ \& Rhoades B E, Fixed points for set valued functions without continuity, Indian J. Pure Appl. Math. 29 (1998), 227.

[4] Menger K, Statistical metric, Proc. Nat. Acad. (USA) 28 (1942), 535.

[5] Mishra S N, Common fixed points of compatible mappings in probabilistic metric spaces, Math. Japon. 36 (1991), 283.

[6] O'Regan D \& Saadati R, Nonlinear contraction theorems in probabilistic spaces, Appl. Math. Comput. 195 (2008), 86.

[7] Schweizer B \& Sklar A, Probabilistic Metric Spaces, Elsevier, North-Holland, New York, 1983.

[8] Sehgal V M \& Bharucha-Reid A T, Fixed point of contraction mappings on probabilistic metric spaces, Math. System Theory 6 (1972), 97.

[9] Shakeri S, A contraction theorem in Menger probabilistic metric spaces, J. Nonlinear Sci. Appl. 1 (2008), 189.

[10] Singh B \& Jain S, A fixed point theorem in Menger space through weak compatibility, $J$. Math. Anal. Appl. 301 (2005), 439. 